\title{
Chain breaks and the susceptibility of $\operatorname{Sr}_{2} \mathrm{Cu}_{1-x} \mathrm{Pd}_{x} \mathrm{O}_{3+\delta}$ and other doped quasi one-dimensional antiferromagnets
}

\author{
J. Sirker, ${ }^{1}$ N. Laflorencie, ${ }^{1}$ S. Fujimoto, ${ }^{2}$ S. Eggert ${ }^{3}$ and I. Affleck ${ }^{1}$ \\ ${ }^{1}$ Department of Physics and Astronomy, University of British Columbia, Vancouver, British Columbia, Canada V6T 1Z1 \\ ${ }^{2}$ Department of Physics, Kyoto University, Kyoto 606-8502, Japan \\ ${ }^{3}$ Department of Physics, University of Kaiserslautern, D-67663 Kaiserlautern, Germany
}

(Dated: May 25, 2021)

\begin{abstract}
We study the magnetic susceptibility of one-dimensional $S=1 / 2$ antiferromagnets containing non-magnetic impurities which cut the chain into finite segments. For the susceptibility of long anisotropic Heisenberg chain-segments with open boundaries we derive a parameter-free result at low temperatures using field theory methods and the Bethe Ansatz. The analytical result is verified by comparing with Quantum-Monte-Carlo calculations. We then show that the partitioning of the chain into finite segments can explain the Curie-like contribution observed in recent experiments on $\mathrm{Sr}_{2} \mathrm{Cu}_{1-x} \mathrm{Pd}_{x} \mathrm{O}_{3+\delta}$. Possible additional paramagnetic impurities seem to play only a minor role.

PACS numbers: 75.10.Jm, 75.10.Pq, 02.30.Ik
\end{abstract}

Measurements of the magnetic susceptiblity, $\chi$, on antiferromagnetics at low temperatures, $T$, often reveal a (Curie) term $\chi \propto 1 / T$. This is usually associated with paramagnetic impurities (free spins). For onedimensional $\mathrm{S}=1 / 2$ systems, however, a second mechanism exists which leads to a contribution with somewhat similar $T$-dependence. Non-magnetic impurities can cut the chain into finite segments with essentially free boundaries. At $T / J \ll 1 / L$ ( $J$ being the exchange constant) a segment with odd length $L$ will lock into its doublet ground state yielding also a $1 / T$ contribution $[1-4]$. However, the behavior is considerably more complicated at higher $T$. As we show, fitting data to a Curie form for a limited range of $T$ can lead to an underestimate of the impurity concentration by as much as a factor of ten! In general, the different $T$-dependence could make it possible to distinguish this type of impurity from paramagnetic ones providing useful structural information.

These results are relevant for materials like $\mathrm{Sr}_{2} \mathrm{CuO}_{3+\delta}$ which is known to be an almost ideal realization of the spin-1/2 Heisenberg chain with a nearest-neighbor coupling constant $J \sim 2200 \mathrm{~K}$ and a low Néel temperature $T_{N} \sim 5 \mathrm{~K} \sim 0.002 J[5,6]$. Measurements of the magnetic susceptibility have revealed a Curie contribution which could be dramatically reduced by annealing. The Curie term therefore is believed to be mainly caused by excess oxygen $[5,6]$. Recently, susceptibility measurements have also been reported on $\mathrm{Sr}_{2} \mathrm{Cu}_{1-x} \mathrm{Pd}_{x} \mathrm{O}_{3+\delta}$ with $\mathrm{Pd}$ serving as a non-magnetic impurity [7].

In this letter we show that these susceptibility data can be explained by taking the segmentation of the chain due to the non-magnetic impurities into account properly without including any additional paramagnetic impurities. To this end we derive a parameter-free analytical result for the susceptibility of an anisotropic Heisenberg chain with finite length and open boundary conditions (OBCs) at low temperatures using field-theory methods. This extends and generalizes previous results in the scal- ing limit $[2,3]$ and for the boundary susceptibility in the thermodynamic limit [8-11]. We will verify our analytical result using Quantum-Monte-Carlo (QMC) calculations.

We consider an anisotropic spin-1/2 Heisenberg chain consisting of segments with length $L$ and OBCs

$$
H=J \sum_{i=1}^{L-1}\left[S_{i}^{x} S_{i+1}^{x}+S_{i}^{y} S_{i+1}^{y}+\Delta S_{i}^{z} S_{i+1}^{z}\right]
$$

Here, $\Delta$ parameterizes the exchange anisotropy. We assume that $T \gg T_{N}$ so that interchain couplings can be safely ignored. As argued at the end of this letter, longer-range interactions which could bridge between the chain segments can be neglected as well in the parameter regime we are interested in. For a concentration of chain breaks $p$ the average chain length is given by $\bar{L}=1 / p-1$ and the averaged susceptibility by [12]

$$
\chi_{p}=p^{2} \sum_{L} L(1-p)^{L} \chi(L) .
$$

We now calculate the susceptibility $\chi(L)=$ $\left\langle\left(\sum_{i} S_{i}^{z}\right)^{2}\right\rangle /(L T)$ in the low $T$ large $L$ limit using fieldtheory methods. We start with the Hamiltonian (1) in the scaling limit, i.e., ignoring irrelevant operators. The Hamiltonian is then equivalent to a free boson model [13]

$$
H=\frac{v}{2} \int_{0}^{L} d x\left[\Pi^{2}+\left(\partial_{x} \phi\right)^{2}\right] .
$$

Here $\phi$ is a bosonic field obeying the standard commutation rule $\left[\phi(x), \Pi\left(x^{\prime}\right)\right]=\mathrm{i} \delta\left(x-x^{\prime}\right)$ with $\Pi=v^{-1} \partial_{t} \phi$. The velocity $v$ is a known function of the anisotropy $\Delta$. Using the mode expansion for OBCs[2]

$$
\begin{aligned}
\phi(x, t) & =\pi R+2 \pi R S_{z} \frac{x}{L} \\
& +\sum_{n=1}^{\infty} \frac{\sin (\pi n x / L)}{\sqrt{\pi n}}\left(\mathrm{e}^{-i \pi n \frac{v t}{L}} a_{n}+\mathrm{e}^{i \pi n \frac{v t}{L}} a_{n}^{\dagger}\right)
\end{aligned}
$$


where $R$ is the compactification radius of the bosonic field, the Hamiltonian (3) can also be expressed as

$$
H=\frac{\pi v}{K L} S_{z}^{2}-h S_{z}+\frac{\pi v}{L} \sum_{n=1}^{\infty} n\left(a_{n}^{\dagger} a_{n}+1 / 2\right) .
$$

Here $a_{n}$ is a bosonic annihilation operator, $S_{z}$ an integer (half-integer) for $L$ even (odd) and $h$ the magnetic field. $K$ - the so called Luttinger parameter - is related to the compactification radius by $K=1 /\left(2 \pi R^{2}\right)$ and is a known function of anisotropy $\Delta$ as well. The susceptibility in the scaling limit is then given by

$$
\chi_{s}=-\left.\frac{\partial^{2} f}{\partial h^{2}}\right|_{h=0}=\frac{1}{L T} \frac{\sum_{S_{z}} S_{z}^{2} \exp \left[-\frac{\pi v}{K L T} S_{z}^{2}\right]}{\sum_{S_{z}} \exp \left[-\frac{\pi v}{K L T} S_{z}^{2}\right]} .
$$

For $L T / v \rightarrow 0$ and $L$ even $\chi_{s} \sim \frac{2}{L T} \exp \left[-\frac{\pi v}{K L T}\right]$ whereas for $L$ odd $\chi_{s} \sim(4 L T)^{-1}$. For $L T / v \rightarrow \infty$ the thermodynamic limit result $\chi_{s}=K /(2 \pi v)$ is recovered. Corrections to scaling occur due to irrelevant bulk and boundary operators. The leading bulk irrelevant operator for $0<\Delta \leq 1$ is due to Umklapp scattering yielding the following correction to $(3)$

$$
\delta H=\lambda_{1} \int_{0}^{L} d x \cos (2 \phi / R)
$$

with $\lambda_{1}$ being the Umklapp scattering amplitude. To obtain $\chi$ to first order in the operator (7) for $L$ and $T$ finite the expectation value $\langle\exp ( \pm 2 \mathrm{i} \phi / R)\rangle$ has to be calculated. Using the mode expansion (4) this expectation value splits into an $S_{z}$ (zero mode) and an oscillator part. Upon using the cumulant theorem for bosonic modes we obtain $\langle\exp ( \pm 2 \mathrm{i} \phi / R)\rangle=$ $\langle\exp ( \pm 2 \mathrm{i} \phi / R)\rangle_{S_{z}} \exp \left(-2\langle\phi \phi\rangle_{\text {osc. }} / R^{2}\right)$ with

$$
\langle\phi \phi\rangle_{\text {osc. }}=\sum_{l=1}^{\infty} \frac{\sin ^{2}(\pi l x / L)}{\pi l}\left(1+\frac{2}{\mathrm{e}^{\pi v l /(T L)}-1}\right) .
$$

Introducing a cutoff $\alpha$ for the zero temperature part in (8) and using $\sum_{l=1}^{\infty} z^{l} / l=-\ln (1-z)$ for $|z|<1$ we obtain the following correction to $(6)$

$$
\begin{aligned}
\delta \chi_{1} & =\frac{2 \tilde{\lambda}_{1}}{T^{2}}\left(\frac{\pi}{L}\right)^{2 K} \eta^{6 K}\left(\mathrm{e}^{-\frac{\pi v}{T L}}\right) \int_{0}^{1 / 2} d y \frac{g_{0}\left(y, \mathrm{e}^{-\frac{\pi v}{K L T}}\right)}{\theta_{1}^{2 K}\left(\pi y, \mathrm{e}^{-\frac{\pi v}{2 T L}}\right)} \\
& \times 2^{2 K} \sin ^{2 K}(\pi y)\left[\left(1-\mathrm{e}^{2 \pi \mathrm{i} y} \mathrm{e}^{-\alpha \pi / L}\right) \times \text { h.c. }\right]^{-K}(9)
\end{aligned}
$$

with

$$
\begin{aligned}
g_{0}(y, q) & =-\frac{\sum_{S_{z}} S_{z}^{2} \cos \left(4 \pi S_{z} y\right) q^{S_{z}^{2}}}{\sum_{S_{z}} q^{S_{z}^{2}}} \\
& +\frac{\left(\sum_{S_{z}} \cos \left(4 \pi S_{z} y\right) q^{S_{z}^{2}}\right)\left(\sum_{S_{z}} S_{z}^{2} q^{S_{z}^{2}}\right)}{\left(\sum_{S_{z}} q^{S_{z}^{2}}\right)^{2}}
\end{aligned}
$$

Here $\eta(x)$ is the Dedekind eta-function, $\theta_{1}(u, q)$ the elliptic theta function of the first kind [14] and $\tilde{\lambda}_{1}=\alpha^{2 K} \lambda_{1}$.
For $K<3 / 2$ the integral in (9) is convergent, the cutoff can be dropped, and the last line becomes equal to one. An additional correction arises due to the presence of an irrelevant boundary operator. In essence, this operator changes the length of the chain $L$ to some effective length $L+a[2]$. Replacing $L$ by $L+a$ in the exponentials in (6) and expanding to lowest order in $a$ yields

$$
\delta \chi_{2}=-\pi v a g_{1}(\exp [-\pi v /(K L T)]) /\left(K T^{2} L^{3}\right)
$$

with

$$
g_{1}(q)=\frac{\left(\sum_{S_{z}} S_{z}^{2} q^{S_{z}^{2}}\right)^{2}}{\left(\sum_{S_{z}} q^{S_{z}^{2}}\right)^{2}}-\frac{\sum_{S_{z}} S_{z}^{4} q^{S_{z}^{2}}}{\sum_{S_{z}} q^{S_{z}^{2}}}
$$

For $K>3 / 2$ we isolate the cutoff dependence by subtracting the Taylor expand of the integrand in (9) to first non-vanishing order in $y$, and then take $\alpha \rightarrow 0$, yielding a convergent integral. The cutoff dependent part has exactly the same form as (11). We can therefore think of $a$ in (11) as a parameter incorporating both contributions. In the thermodynamic limit $g_{1}\left(\mathrm{e}^{-\pi v /(K L T)}\right) \rightarrow$ $K^{2} T^{2} L^{2} /\left(2 \pi^{2} v^{2}\right)$ and $\delta \chi_{2} \rightarrow K a /(2 \pi v L)$. In this limit we can compare the field theory result with a recent calculation of the boundary susceptibility based on the Bethe ansatz $[9,10]$ leading to

$$
a=2^{-1 / 2} \sin [\pi K /(4 K-4)] / \cos [\pi /(4 K-4)] .
$$

Because the amplitude $\tilde{\lambda}_{1}$ is known exactly as well $[10,15]$, the obtained result is parameter-free. In Fig. 1 a comparison between this field theory result and QMC data for $\Delta=3 / 4$ is shown and in the left inset the corrections to the scaling limit (6) due to the leading irrelevant bulk and boundary operator are visualized. More generally, we always find excellent agreement if $T / J \lesssim 0.1$ and $L \gtrsim 10$. The range of validity can be extended in principle by including terms in higher order perturbation theory in $\lambda_{1}$. However, if $T / v \gg 1 / L$ it is justified to separate $\chi$ into a bulk, $\chi_{\text {bulk }}$, and a boundary contribution, $\chi_{B}$, and to perform the thermodynamic limit for each of them separately. A combination of this result [10] (blue dashed line in Fig. 1) with the result derived in this letter yields an excellent parameter-free description of the susceptibility for anisotropic chains of finite length up to $T \sim J / 2$

For $K=1(\Delta=1)$ perturbation theory alone is not sufficient because Umklapp scattering becomes marginal. In this case the coupling constant $\tilde{\lambda}_{1}$ has to be replaced by a renormalization group (RG) improved running coupling constant $[15,16] . \quad \tilde{\lambda}_{1}$ then becomes a function of the two length scales $L$ and $v / T$. For small enough energies the smaller scale will always dominate and the running coupling constant becomes $\tilde{\lambda}_{1}(L, v / T)=g / 4$ with [15]

$$
1 / g+\ln (g) / 2=\ln \left(\sqrt{2 / \pi} e^{1 / 4+\gamma} \min [L, v / T]\right)
$$




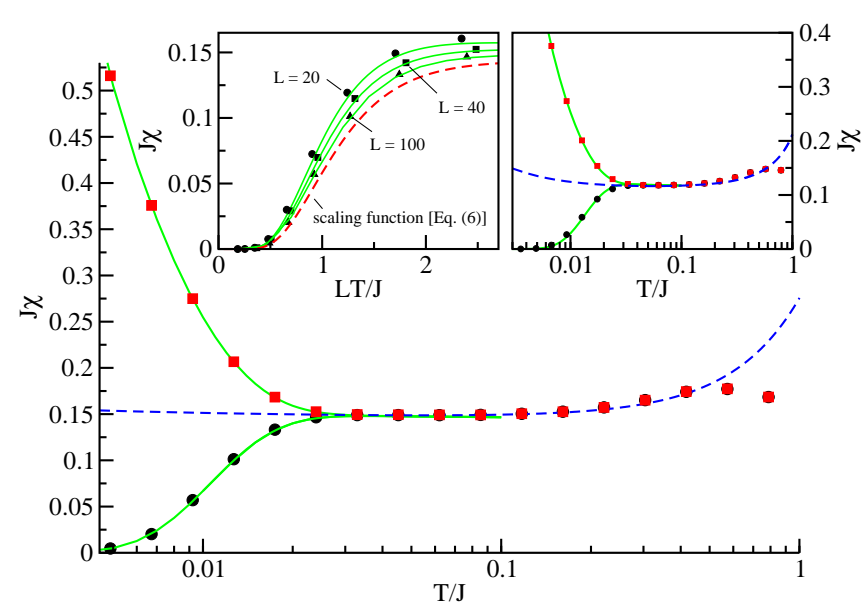

FIG. 1: (Color online) QMC data for $\Delta=3 / 4$ and $L=99$ (red squares), $L=100$ (black dots) compared to the field theory result derived here (green solid lines). The blue dashed line denotes $\chi=\chi_{\text {bulk }}+\chi_{B} / L$ (see Eqs. $(148,149)$ in [10]). Left inset: Corrections to scaling for even length chains and $\Delta=3 / 4$. QMC data are represented by symbols, the field theory by the green lines. Right inset: Same as main figure for $\Delta=1$ with $a=5.8$ and $\chi_{\text {bulk }}, \chi_{B}$ as obtained in $[8,15]$.

where $\gamma$ is the Euler constant. $\delta \chi_{1}$ in the isotropic case is therefore given by (9) with the last line dropped and $\tilde{\lambda}_{1}$ replaced by $\tilde{\lambda}_{1}(L, v / T)$. Furthermore, $K$ in $(6)$ should be replaced by $1+g(L, T) / 2$ so that $K L$ in the exponentials gets replaced by $L(1+g / 2+a / L)$. For this case $a$ is not known and we will use it as a fitting parameter. This way we obtain excellent agreement if $T / J \lesssim 0.1$ and $L \gtrsim 10$ (see right inset of Fig. 1). At $L T \ll v$ the corrections to the scaling formula (6) in the isotropic case due to the marginal interaction can also be understood as follows. In this limit the most important correction to (6) arises from the correction to the excitation energy of the lowest excited states with $S^{z}= \pm 1, E \rightarrow(\pi v / L)[1-g(L)]$, [17] corresponding to the replacement $1 / K \rightarrow 1-g(L)$ in $(6)$. Here $g(L)$ is given by (14) but with $\min [L, v / T]$ simply replaced by $L$.

These analytical results, supplemented by numerical data for $L \lesssim 10$, can be used to calculate the averaged susceptibility (2) at low temperatures. In addition, we have calculated $\chi(L)$ numerically for all $L \in[1,100]$ and certain additional $L$ up to $L=1000$ at various temperatures. An interpolation and extrapolation was then used to obtain $\chi(L)$ for all lengths up to $L=5000$ at a given temperature [19]. This allows us to reliably calculate $\chi_{p}$ (2) for impurity concentrations $p \gtrsim 0.2 \%(\bar{L} \lesssim 500)$.

Experimental data for the susceptibility of the quasi one-dimensional compounds $\mathrm{Sr}_{2} \mathrm{Cu}_{1-x} \mathrm{Pd}_{x} \mathrm{O}_{3+\delta}$ have been analyzed in [5-7] by decomposing it into $\chi=$ $\chi_{0}+\chi_{C}+\chi_{\text {inf. }}$. Here $\chi_{0}$ represents a constant part due to core diamagnetism and Van Vleck paramagnetism, $\chi_{C}$ a Curie term and $\chi_{\text {inf. }}$ the susceptibility (per unit length) of the infinite $S=1 / 2$ Heisenberg chain. However, our

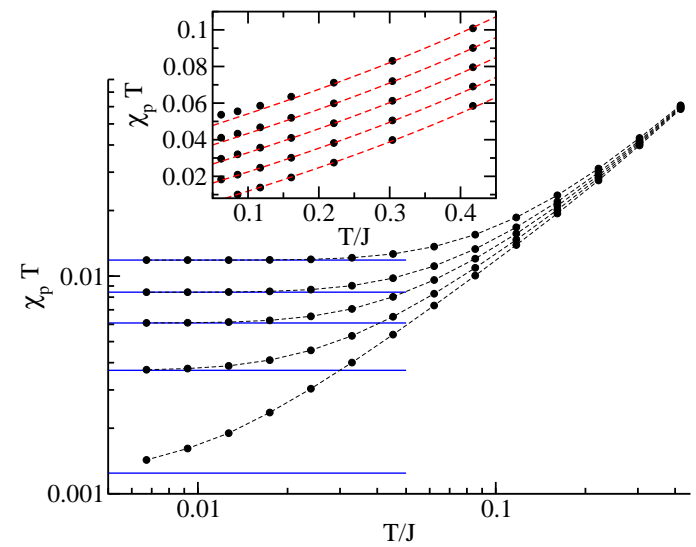

FIG. 2: (Color online) $T \chi_{p}$ for $p=0.01,0.03,0.05,0.07,0.1$ from bottom to top (black dots with dashed lines being a guide to the eye). The blue solid lines denote the value $(p / 4)$. $(1-p) /(2-p)$ reached in the limit $T \ll p v$. Inset: Same data for $T \chi_{p}$ with subsequent curves shifted by 0.01 compared to $T \chi_{\text {bulk }}+p /[12 \ln (2.9 J / T)]$ (red dashed lines) reached in the opposite limit.

results show that non-magnetic impurities (chain breaks) have a quite different effect than paramagnetic impurities. In the extreme low- $T$ limit, $T \ll p v$, chain breaks will lead to $1 / T$-behavior with a strength reduced from that which would arise from the same density of paramagnetic impurities by the fraction of chains of odd length, $(1-p) /(2-p) \approx 1 / 2$. In the opposite limit $p v \ll T$ (but $T$ still $\ll J$ ), chain breaks lead to a correction to the pure result of $\approx p /[12 T \ln (2.9 J / T)]$ corresponding to an "effective paramagnetic impurity concentration" of $\approx p /[3 \ln (2.9 J / T)]$ which can be $\ll p$ for $T \ll J$. This behavior is illustrated in Fig. 2.

It has been shown that the Curie-like contribution to the susceptibility of $\mathrm{Sr}_{2} \mathrm{CuO}_{3+\delta}$ can be significantly reduced by annealing under Ar atmosphere indicating that this contribution is mostly due to excess oxygen [20]. We expect the additional interstitial oxygen ions to be in a $\mathrm{O}^{2-}$ ionization state. Each oxygen ion would then dope two holes into the chains. If these holes are immobile they effectively act like chain breaks. It has been pointed out that the next-nearest neighbor coupling is not that small, $J_{2} \sim J / 16 \approx 140 \mathrm{~K}$ [18]. Whereas we expect this irrelevant coupling to cause only small corrections to the susceptibility of an isolated chain segment the fact that it can bridge a chain break could be significant. A first order perturbative calculation, however, shows that the correction to the averaged susceptibility is given by $\delta \chi_{p} \sim p J_{2} \chi_{p}^{2}$. We can therefore ignore both interactions as long as $T \gg \max \left(p J_{2}, J_{\perp}\right)$ making the model discussed here applicable. As shown in the inset of Fig. 3 it is indeed possible to explain the data in [5] for the unannealed sample using a $0.6 \%$ concentration of chain breaks. If our picture is correct the amount of excess oxygen is significantly larger than assumed in [5], 0.06\%. 


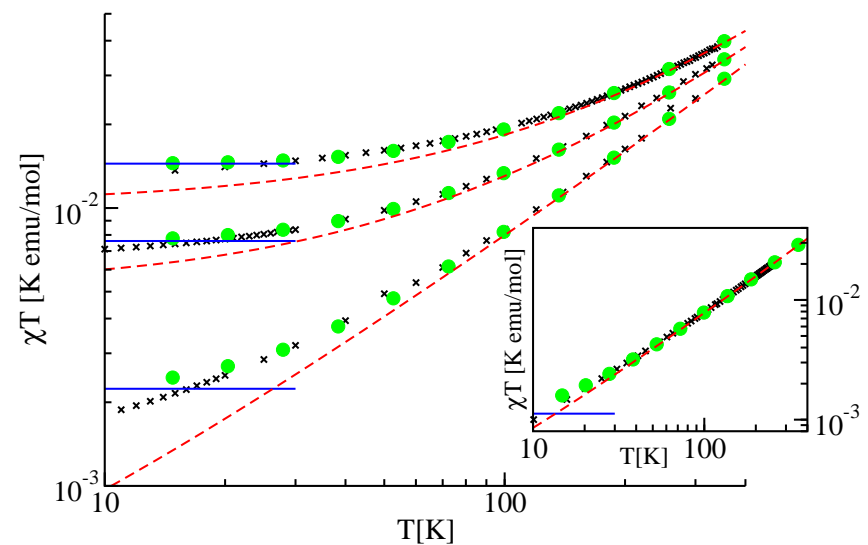

FIG. 3: (Color online) Measured susceptibility $T\left(\chi-\chi_{0}\right)$ for $\mathrm{Sr}_{2} \mathrm{Cu}_{1-x} \mathrm{Pd}_{x} \mathrm{O}_{3+\delta}$ with $x=0.5,1,3 \%$ (crosses from bottom to top) where a constant $\chi_{0}$ (see Table I) has been subtracted. The green dots represent the best theoretical fit with $p$ as in Table I. Subsequent curves are shifted by $3 \times 10^{-3}$. The blue solid lines represent $(p / 4) \cdot(1-p) /(2-p)$ and the red dashed lines $T \chi_{\text {bulk }}+p /[12 \ln (2.9 J / T)]$. Note that fits $\chi=$ $\chi_{0}+\chi_{\text {inf. }}+p_{\text {para }} /(4 T)$ in the regime $T>50 \mathrm{~K}$ would be similar to the red dashed lines with effective paramagnetic impurity concentrations $p_{\text {para }} \approx p /[3 \ln (2.9 J / T)]$ an order of magnitude smaller than the nominal $\mathrm{Pd}$ concentrations. Inset: Same for "as grown" sample of $\mathrm{Sr}_{2} \mathrm{CuO}_{3+\delta}$ from Ref. [5].

The $\mathrm{Sr}_{2} \mathrm{Cu}_{1-x} \mathrm{Pd}_{x} \mathrm{O}_{3+\delta}$ samples used in [7] were not annealed. This complicates the analysis of the susceptibility data because excess oxygen again leads to chain breaks in addition to those caused by the non-magnetic $\mathrm{Pd}$ ions. The amount of excess oxygen hereby seems to vary significantly from sample to sample. This is most apparent when comparing the measured susceptibilities for $x=0.5 \%$ and $x=1 \%$ which are almost identical apart from a small constant shift. We therefore treat the impurity concentration $p$ as a fitting parameter. Results where we used $J=2200 \mathrm{~K}$ and a gyromagnetic factor $g=2$ are shown in Fig. 3 and Table I. The figure clearly

TABLE I: Concentration $x$ of $\mathrm{Pd}$ ions in experiment compared to impurity concentration $p$ and constant contribution $\chi_{0}$ yielding the best theoretical fit. The first line corresponds to the "as grown" sample of $\mathrm{Sr}_{2} \mathrm{CuO}_{3+\delta}$ from Ref. [5].

\begin{tabular}{ccc}
\hline \hline$x$ (Exp.) & $p$ (Theory) & $\chi_{0}[\mathrm{emu} / \mathrm{mol}]$ \\
\hline 0.0 & 0.006 & $-7.42 \times 10^{-5}$ \\
0.005 & 0.012 & $-7.7 \times 10^{-5}$ \\
0.01 & 0.014 & $-7.5 \times 10^{-5}$ \\
0.03 & 0.024 & $-7.5 \times 10^{-5}$ \\
\hline \hline
\end{tabular}

shows that the susceptibility data are indeed quite different from what would be expected for paramagnetic impurities. Most of the data are taken in the non-trivial crossover regime $p v \sim T$ and are well described by our theory. Note, that the deviations at low temperatures are expected because the suppression of the susceptibility due to interchain and next-nearest neighbor interactions is not taken into account in our model. For $x=0.005$ and 0.01 the effective impurity concentrations $p$ are larger than the nominal $\mathrm{Pd}$ concentration. $p-x$ roughly coincides with the number of chain breaks in the "as grown" $\mathrm{Sr}_{2} \mathrm{CuO}_{3+\delta}$ supporting our picture of additional chain breaks due to excess oxygen. For $x=0.03$, however, $p$ is smaller than the nominal $\mathrm{Pd}$ concentration. Possible explanations are that for larger doping levels some of the $\mathrm{Pd}$ ions go in interstitially instead of replacing $\mathrm{Cu}$ ions or that the sample exhibits some sort of phase separation. Future measurements on annealed $\mathrm{Sr}_{2} \mathrm{Cu}_{1-x} \mathrm{Pd}_{x} \mathrm{O}_{3}$ samples would be helpful to clarify some of these issues.

The authors acknowledge valuable discussions with W. N. Hardy, K. M. Kojima and G. A. Sawatzky. This research was supported by NSERC (J.S., N.L. I.A.), the DFG (J.S.) and the CIAR (I.A.). Numerical simulations have been performed on the Westgrid network.

[1] J. C. Bonner and M. E. Fisher, Phys. Rev. 135, A640 (1964).

[2] S. Eggert and I. Affleck, Phys. Rev. B 46, 10866 (1992).

[3] S. Wessel and S. Haas, Phys. Rev. B 61, 15262 (2000).

[4] B. Schmidt, et al., Eur. Phys. J. B 32, 43 (2003).

[5] N. Motoyama, H. Eisaki, and S. Uchida, Phys. Rev. Lett. 76, 3212 (1996).

[6] T. Ami, et al., Phys. Rev. B 51, 5994 (1995).

[7] K. M. Kojima, et al., Phys. Rev. B 70, 094402 (2004).

[8] S. Fujimoto and S. Eggert, Phys. Rev. Lett. 92, 037206 (2004).

[9] M. Bortz and J. Sirker, J. Phys. A: Math. Gen. 38, 5957 (2005).

[10] J. Sirker and M. Bortz, J. Stat. Mech. P01007 (2006).

[11] A. Furusaki and T. Hikihara, Phys. Rev. B 69, 094429 (2004).

[12] S. Eggert, I. Affleck, and M. D. P. Horton, Phys. Rev. Lett. 89, 047202 (2002).

[13] I. Affleck, Fields, Strings and Critical Phenomena (Les Houches, Session XLIX, Amsterdam, 1988), p. 563, edited by E. Brézin and J. Zinn-Justin.

[14] I. Gradshteyn and I. Ryzhik, Table of Integral, Series, and Products (Academic Press, New York, 2000).

[15] S. Lukyanov, Nucl. Phys. B 522, 533 (1998).

[16] I. Affleck, D. Gepner, H. J. Schulz, and T. Ziman, J. Phys. A 22, 511 (1989).

[17] S. Qin and I. Affleck, J. Phys. A 32, 7815 (1999).

[18] H. Rosner, et al., Phys. Rev. B 56, 3402 (1997).

[19] See EPAPS Document No. [] for the QMC as well as the interpolated and extrapolated data.

[20] From annealed samples we know that the true paramagnetic impurity concentration is $0.013 \%$ at most [5] and can therefore be neglected in the following. 